\title{
Predictors of peritonitis in peritoneal dialysis: experience during 3 decades
}

David Fiel, Joana Santos, Rita Vicente, Iolanda Santos, Beatriz Malvar, Rui Silva, Manuel Amoedo, Carlos Pires

Division of Nephrology, Hospital do Espírito Santo, Évora, Portugal

\section{ABSTRACT}

Introduction: Peritonitis is a common complication in peritoneal dialysis patients and a major cause for dropout. Systematic report and frequent trend analysis are thought to be major drivers for improvement in this area. The authors analysed peritonitis outcomes dating back over 20 years in a peritoneal dialysis unit of a central hospital. Methods: Retrospective study from 1993 to 2018: assessment of demographic, clinical and microbiological profiles of patients with peritonitis. We performed univariate and multivariate analysis (multiple logistic regression) to predict peritonitis risk factors, and survival analysis (Cox proportional hazards model) to determine the impact on outcomes (mortality and technique survival). Results: We included 225 patients, average age of $48.3 \pm 14.7$ years, average time of follow-up of $38 \pm 25$ months, with a total of 221 episodes of peritonitis ( 0.31 peritonitis/patient.year), 76\% resulting in cure. Most frequent agents were Staphylococci: Coagulase-Negative (23\%) and Staphylococcus aureus (19\%, of which $24 \%$ were methicillin-resistant). Gram-negative infections led to higher rate of catheter removal, transfer to hemodialysis or death ( $49 \%$ of cases vs. $17 \%$ in Gram-positive). Primary end-point was death or transfer to hemodialysis, with a median time of $94(\min 4, \max 94)$ and $66(\min 3, \max 105)$ months, respectively. The occurrence of at least one peritonitis was the major variable that influenced transfer to hemodialysis (OR 3.94 [2.12 - 7.58], $p<0.001 *$, whereas an event in the first year also negatively affected the time to dialysis technique switch (median time 38.7 [26.2 - 58.4] vs. $67.8[59.5-80.3]$ months, log-rank = 0.02*), but without impact on mortality. Only the peritoneal dialysis modality (OR: automated peritoneal dialysis vs. continuous ambulatory peritoneal dialysis 0.38 [0.19-0.74]) was predictive of peritonitis in multivariate analysis. Conclusions: In this single-center long-term analysis, where the rate of peritonitis was within recommended values, automated peritoneal dialysis seemed to have a protective impact. The number of peritonitis and peritonitis occurrence during the first year worsened technique survival, emphasizing early peritonitis prevention.

Keywords: Automated Peritoneal Dialysis; Continuous Ambulatory Peritoneal Dialysis; Peritonitis

\section{INTRODUCTION}

Peritoneal dialysis (PD) is one of the available treatments for end-stage renal disease (ESRD) with some advantages when compared to its counterpart hemodialysis (HD), namely, more autonomous and self-governing treatment, absence of a vascular access and its cannulation, no need for anticoagulation and even less mortality in the first years of treatment, as shown by Fenton et al. ${ }^{1}$ However, median time to technique dropout continues to be lower than expected. Long-term exposition to conventional peritoneal dialysis solutions have been associated with an impairment in host cell defense, reduction in peritoneal mesothelial cell viability, and progressive peritoneal membrane injury and function loss. ${ }^{2}$ Furthermore, peritonitis is a common complication in patients undergoing PD and still a major cause of patient mortality and technique dropout. ${ }^{3-6}$ According to the ISPD Peritonitis Recommendations 2016, peritonitis is the direct or major contributing cause of death in around $16 \%$ of PD patients and may cause structural and functional alterations of the peritoneal membrane, eventually leading to membrane failure. ${ }^{7}$ Reported rates of PD peritonitis range widely from 0.06 to 1.66 episodes per patient-year across different centers and countries. ${ }^{2}$
We are presenting the results of a retrospective study, designed to disclose peritonitis epidemiology, risk factors and outcomes dating back over 20 years in a PD unit of a central hospital in Portugal.

\section{METHODS}

\section{General design}

Following a retrospective, observational design, we assessed demographic, clinical and microbiological profiles of patients with peritonitis undergoing chronic PD in our center during the period between 1993 and 2018. The authors defined 2 main study variables: total number of peritonitis and peritonitis in the first year of PD. The main outcome variable was time until death or transfer to HD.

\section{Study population}

This study included all prevalent patients (under treatment for at least 3 months), older than 18 years who started PD therapy in our 
center from the 1st January 1993 until the 31st December 2016 and follow-up until 31st December 2018.

\section{Study variables}

Demographic, clinical and microbiological profiles of patients with peritonitis were assessed by recovering data from the hospital's patient clinical database and from our PD unit patient local registry. The main study variables were $I$. the total number of peritonitis $(0$, $1,2+)$ and II. any episode of peritonitis in the first year of PD. The main outcome variable was time until death or transfer to HD (primary). We defined peritonitis according to standard ISPD rules. ${ }^{7}$ We recorded the main clinical and demographic variables with a potential association with clinical outcomes, including the risk of peritoneal infection (Table I).

\section{Table I}

Characteristics of the study population.

\begin{tabular}{|c|c|}
\hline Variables & Study Group ( $n=225$ ) \\
\hline Female gender, n (\%) & $92(41)$ \\
\hline $\begin{array}{l}\text { Age, years } \\
\quad \text { range } \\
\text { mean } \pm S D\end{array}$ & $\begin{array}{c}19-88 \\
48.3 \pm 14.7\end{array}$ \\
\hline $\begin{array}{l}\text { CKD etiology, } \mathrm{n}(\%) \\
\text { chronic GN } \\
\text { diabetes mellitus } \\
\text { hypertension } \\
\text { interstitial nephritis } \\
\text { other }\end{array}$ & $\begin{array}{l}57(25) \\
55(24) \\
24(11) \\
11(5) \\
78(35)\end{array}$ \\
\hline Diabetes mellitus, $n$ (\%) & $60(27)$ \\
\hline $\begin{array}{l}\text { Follow-up, months } \\
\text { minimum } \\
\text { maximum } \\
\text { mean } \pm S D\end{array}$ & $\begin{array}{c}3 \\
105 \\
38 \pm 25\end{array}$ \\
\hline $\begin{array}{l}\text { PD initiation period, } \mathrm{n}(\%) \\
\begin{array}{c}1993-2004 \\
2005-2018\end{array}\end{array}$ & $\begin{array}{c}91(40) \\
134(60)\end{array}$ \\
\hline $\begin{array}{l}\text { Number of peritonitis, } n(\%) \\
0 \\
1 \\
2+\end{array}$ & $\begin{array}{l}101(45) \\
63(28) \\
61(27)\end{array}$ \\
\hline PD modality, $n(\%)$ & \\
\hline CAPD & $160(71)$ \\
\hline APD & $65(29)$ \\
\hline $\begin{array}{l}\text { Time in } \mathrm{PD} \text {, months } \\
\text { range } \\
\text { mean } \pm S D\end{array}$ & $\begin{array}{c}3-105 \\
37.9 \pm 25.2\end{array}$ \\
\hline $\begin{array}{l}\text { Status at the end of follow-up, } n(\%) \\
\text { active } \\
\text { deceased } \\
\text { transferred to HD } \\
\text { transplanted } \\
\text { recovery of renal function } \\
\text { loss of follow-up }\end{array}$ & $\begin{array}{l}26(12) \\
53(24) \\
82(36) \\
59(26) \\
4(2) \\
1(<1)\end{array}$ \\
\hline
\end{tabular}

APD - Automated Peritoneal Dialysis; CKD - Chronic Kidney Disease; GN - Glomerulonephritis; HD - Hemodialysis; PD - Peritoneal Dialysis; CAPD - Continuous Ambulatory Peritoneal Dialysis; SD Standard Deviation

\section{Strategy of analysis and statistics}

Primary univariate comparisons were produced using multiple logistic regression to predict peritonitis risk factors. Multivariate survival analysis was produced by the Cox proportional hazards model, controlling for other demographic, clinical or laboratory variables with an impact on the incidence of the outcome variables (mortality and technique survival). The R project ${ }^{\circledR}$ software was used for data management, with a significance level of $5 \%$.

\section{RESULTS}

\section{Population overview}

We included 225 patients for analysis. The characteristics of the study population are represented in Table I. The average age was $48.3 \pm 14.7$ years, with a predominance of the male gender $(n=133$, $59 \%$ ). Most patients (71\%) were undergoing continuous ambulatory peritoneal dialysis (CAPD) and $29 \%$ were being treated with automated peritoneal dialysis (APD) at the time of the peritonitis event. Average time of follow-up was $38 \pm 25$ months. Regarding the episodes of peritonitis, 221 events were registered in a total of 124 patients $(0.31$ peritonitis/patient.year), $76 \%$ resulting in cure.

\section{Etiological peritonitis agents and outcomes}

The results of the peritonitis etiological agents are depicted in Table II. The most frequent isolated agents in the peritoneal dwell were Staphylococci: Coagulase-Negative Staphylococci in 23\% of the cases and Staphylococcus aureus in 19\% (of which 24\% were methicillin-resistant Staphylococcus aureus - MRSA). Pseudomonas and fungi are the most aggressive agents and their infection led to either removal of the peritoneal catheter, transfer to HD or death in $100 \%$ of cases. Gram-negative bacterial infections other than Pseudomonas, had a similar rate of cure as Gram-positive (79 vs. $83 \%, p=0.58, n s, q u{ }^{2}$ ).

Differential analysis of epidemiological agents per decade of the studied period are represented in Graphic 1, where we can observe a trend towards an increase in relative frequency of Gram-negative-related peritonitis.

\section{Predictors of peritonitis}

\section{Predictors of Peritonitis}

Univariate analysis of possible predictors of peritonitis is presented in Table III. Patient characteristics such as the higher age $(p=0.03)$, etiology of chronic kidney disease (CKD) $(p=0.012)$ and PD modality $(p<0.001)$ seem to be statistically significant as predictors of peritonitis. However, when considering those variables in a multivariate model, only PD modality (OR: APD vs. CAPD 0.38 [0.19-0.74]) was predictor of peritonitis (Graphic 2).

\section{Outcomes}

Primary end-points were occurrence of death or transfer to hemodialysis which occurred in 53 (24\%) and 82 (36\%) patients 


\section{Table II}

Peritonitis etiological agents and outcomes.

\begin{tabular}{|c|c|c|c|c|c|}
\hline \multirow[b]{2}{*}{ Agents } & \multirow[b]{2}{*}{$n(\%)$} & \multicolumn{4}{|c|}{ Outcome } \\
\hline & & Cure, n (\%) & $\begin{array}{c}\text { Tenckhoff } \\
\text { removal, } \mathbf{n}(\%)\end{array}$ & $\begin{array}{c}\text { Transfer } \\
\text { to HD, } n(\%)\end{array}$ & Death, $n(\%)$ \\
\hline Negative culture & $33(15)$ & 32 (97) & $0(0)$ & $1(3)$ & $0(0)$ \\
\hline Gram + & $134(61)$ & $111(83)$ & $11(8)$ & $8(6)$ & $4(3)$ \\
\hline CNS & $50(23)$ & $46(92)$ & $1(2)$ & $2(4)$ & $1(2)$ \\
\hline Staph. aureus & $42(19)$ & $26(62)$ & $8(19)$ & $5(12)$ & $3(7)$ \\
\hline MSSA & $32(14)$ & $20(62)$ & $7(22)$ & $3(10)$ & $2(6)$ \\
\hline MRSA & $10(5)$ & $6(60)$ & $1(10)$ & $2(20)$ & $1(10)$ \\
\hline Streptococcus & $34(15)$ & $33(97)$ & 0 & $1(3)$ & 0 \\
\hline Other & $8(4)$ & $6(75)$ & $2(25)$ & 0 & 0 \\
\hline Gram - & $51(23)$ & $26(51)$ & $11(22)$ & $13(25)$ & $1(2)$ \\
\hline E. coli & $16(7)$ & $12(75)$ & 0 & $3(19)$ & $1(6)$ \\
\hline Pseudomonas & $18(8)$ & 0 & $9(50)$ & $9(50)$ & 0 \\
\hline Other & $17(8)$ & $14(82)$ & $2(12)$ & $1(6)$ & 0 \\
\hline Fungi & $2(1)$ & 0 & $1(50)$ & $1(50)$ & 0 \\
\hline Polymicrobial & $1(<1)$ & 0 & 0 & $1(100)$ & 0 \\
\hline Total & $221(100)$ & $169(76)$ & $23(10)$ & $24(11)$ & $5(2)$ \\
\hline
\end{tabular}

CNS - Coagulase-negative staphylococci; HD - Hemodialysis; MRSA - Methicillin-resistant Staphylococcus aureus; MSSA - Methicillin-sensitive Staphylococcus aureus

\section{Table III}

Univariate analysis of possible predictors of peritonitis.

\begin{tabular}{|c|c|c|c|c|}
\hline Variables & With Peritonitis & Without Peritonitis & $p$ & test \\
\hline Age, years (mean $\pm S D$ ) & $50.2 \pm 15.1$ & $45.9 \pm 13.9$ & $0.030 *$ & Stu-t \\
\hline $\begin{array}{l}\text { Gender, } \mathrm{n} \\
\text { Male } \\
\text { Female }\end{array}$ & $\begin{array}{l}70(53 \%) \\
51(59 \%)\end{array}$ & $\begin{array}{l}63(47 \%) \\
38(41 \%)\end{array}$ & 0.37 & $\mathrm{Chi}^{2}$ \\
\hline $\begin{array}{l}\text { Diabetes mellitus, } n \\
\text { Yes } \\
\text { No }\end{array}$ & $\begin{array}{l}33(55 \%) \\
91(55 \%)\end{array}$ & $\begin{array}{l}27(45 \%) \\
74(45 \%)\end{array}$ & 0.98 & $\mathrm{Chi}^{2}$ \\
\hline $\begin{array}{l}\text { PD modality, } n \\
\text { CAPD } \\
\text { APD }\end{array}$ & $\begin{array}{c}101(63 \%) \\
23(36 \%)\end{array}$ & $\begin{array}{l}60(37 \%) \\
41(64 \%)\end{array}$ & $<0.001^{*}$ & $\mathrm{Chi}^{2}$ \\
\hline $\begin{array}{l}\text { PD initiation period, } n \\
\text { 1993-2004 } \\
2005-2016\end{array}$ & $\begin{array}{l}52(57 \%) \\
72(54 \%)\end{array}$ & $\begin{array}{l}39(43 \%) \\
62(46 \%)\end{array}$ & 0.61 & $\mathrm{Chi}^{2}$ \\
\hline $\begin{array}{l}\text { CKD etiology, n } \\
\text { Chronic GN } \\
\text { Diabetes mellitus } \\
\text { Hypertension } \\
\text { Interstitial nephritis } \\
\text { Other }\end{array}$ & $\begin{array}{c}24(42 \% \\
30(55 \%) \\
20(83 \%) \\
7(64 \%) \\
43(55 \%)\end{array}$ & $\begin{array}{c}33(58 \%) \\
25(45 \%) \\
4(17 \%) \\
4(36 \%) \\
35(45 \%)\end{array}$ & $0.012 *$ & $\mathrm{Chi}^{2}$ \\
\hline
\end{tabular}

APD - Automated Peritoneal Dialysis; Chi2 - Chi-squared test; CKD - Chronic Kidney Disease; GN - Glomerulonephritis; PD - Peritoneal Dialysis; CAPD- Continuous Ambulatory Peritoneal Dialysis; SD - Standard Deviation; Stu-t - Student's T-test.

respectively. In the logistic regression model the occurrence of at least one peritonitis did not influence the probability of death (OR $0.55[0.25-1.18], p=0.13, n s)$. On the other hand, the presence of diabetes (OR 5.13 [2.41-11.3], p < 0.001) and aging (OR 1.05 per year [1.03-1.08], $p<0.001$ ) have higher probability of outcome, whereas the era of PD initiation (2005-2016 vs. 1993-2004, OR: 0.20 [0.09-0.42], $p<0.001$ ) shows the opposite tendency. - Graphic
3. On the other hand, peritonitis was the major variable that statistically influenced transfer to HD (OR 3.94 [2.12 - 7.58], $p<0.001 *$ ] - Graphic 4.

When we consider the time to event analysis, the median time to death was 93.9 months [IC95\% 93 - 105] and transfer to HD was 66 months [IC95\% 58-78]. Univariate Kaplan-Meier estimates reveal that 


\section{Graphic 1}

Differential analysis of epidemiological agents per decade of the studied period.

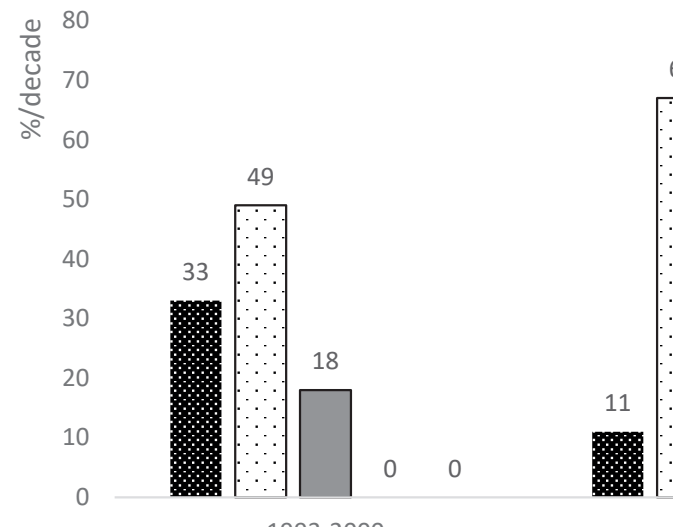

1993-2000

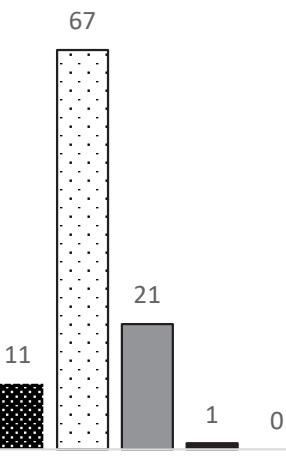

2001-2010

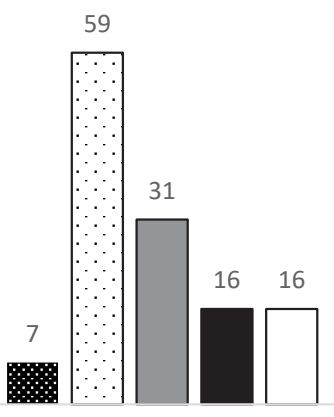

2011-2018

Negative culture $\square$ Gram + $\square$ Gram - —Fungi $\square$ Polymicrobial

\section{Graphic 2}

Multivariate analysis of predictors of peritonitis.

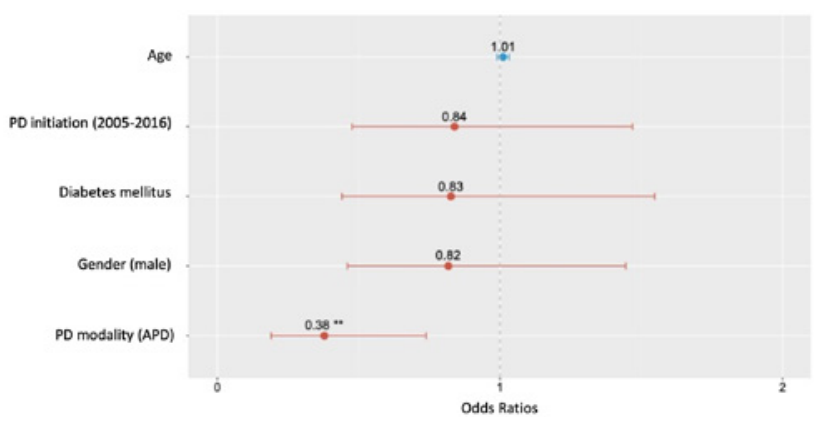

APD - Automated Peritoneal Dialysis; PD - Peritoneal Dialysis.

\section{Graphic 3}

Probability to death.

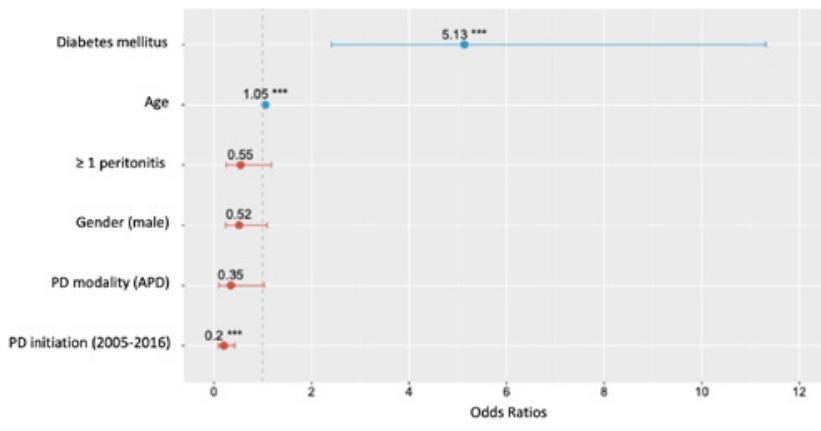

APD - Automated Peritoneal Dialysis; PD - Peritoneal Dialysis.

\section{Graphic 4}

Probability to transfer to HD.

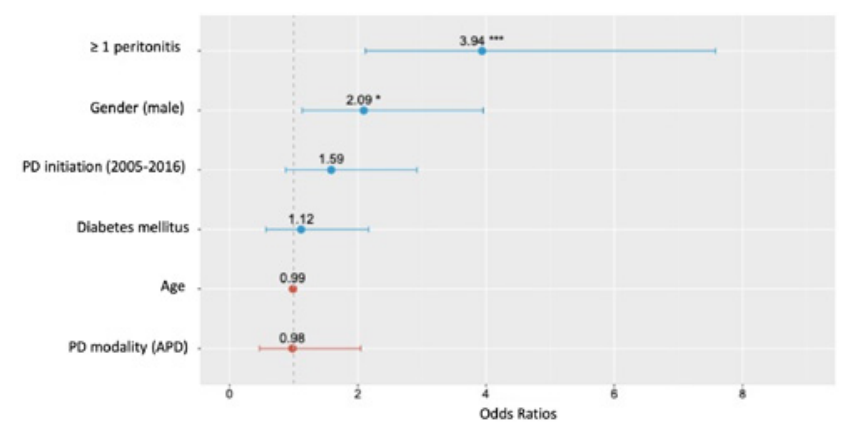

APD - Automated Peritoneal Dialysis; PD - Peritoneal Dialysis.

the occurrence of at least one peritonitis in the first year of treatment does not influence the time to death (log-rank $=0.1$, ns - Graphic 5a.) but indeed affects negatively the time to transfer to HD (median time $38.7[26.2-58.4]$ vs. $67.8[59.5-80.3]$ months, log-rank $=0.02 *$ Graphic 5b).

The multivariate Cox proportional survival model reinforce the results obtained in the logistic regression, as diabetes (HR: 2.6 [1.5 - 4.5], $p<0.001)$, age ((HR: 1.03 per year [1.02 - 1.05], $p<$ $0.001)$ and time of PD initiation (2005- 16 vs. $1993-2004$ HR: 0.38 [0.21 - 0.67], $p<0.001$ ), influence the time to death. As for time to HD transfer, the influence of peritonitis in the first year maintains an important negative trend, although losing its statistical significance (HR: 1.60 [0.92 - 2.75], $p=0.09$, ns), whereas the male gender remains a significant predictor (HR: 1.93 [1.20-3.11], $p=0.007)$. 


\section{Graphic 5}

Differential analysis of epidemiological agents per decade of the studied period.

A. Time to Death

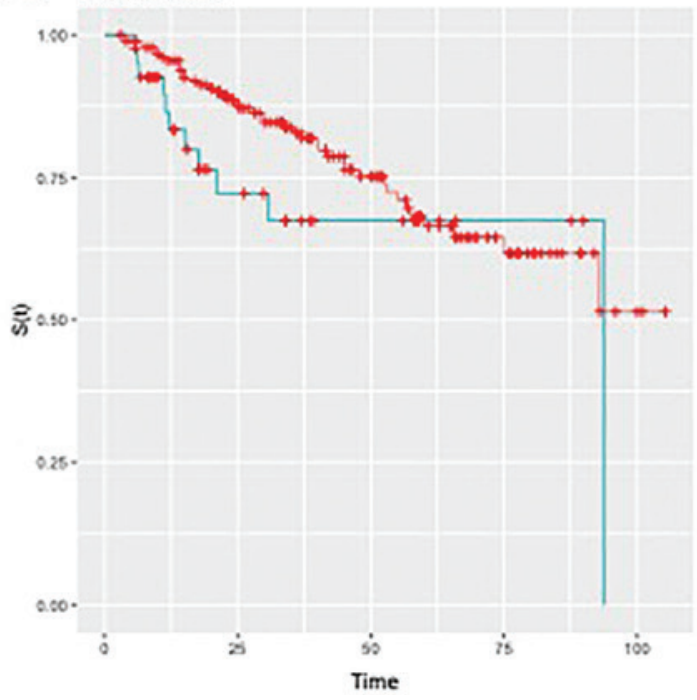

B. Time to HD transfer

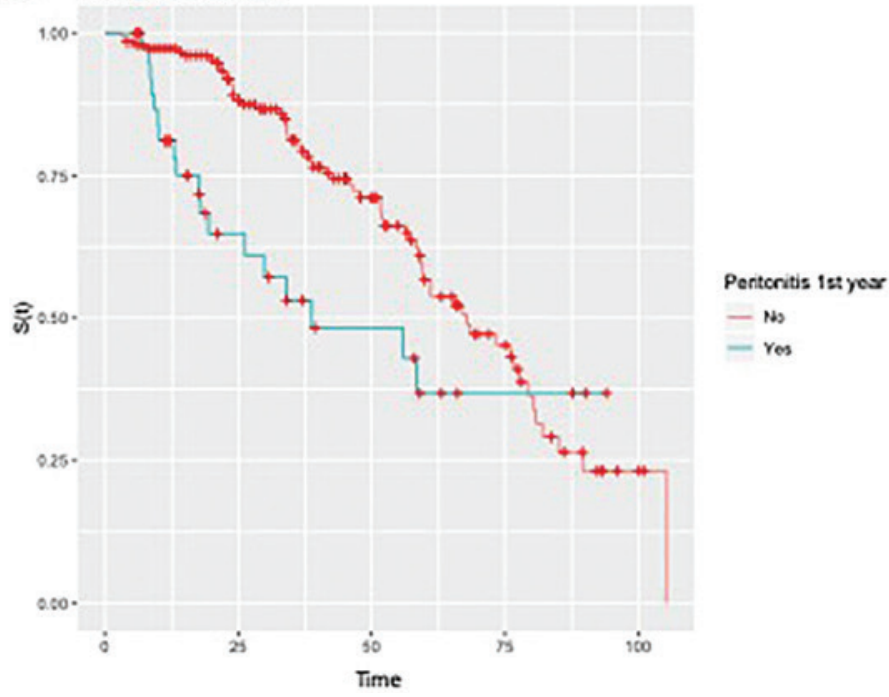

\section{DISCUSSION}

\section{Peritonitis Rate}

In our analysis, the global rate of peritonitis was well within recommended values: 0.31 peritonitis/patient.year. Despite there being a substantial variation in the peritonitis rates reported by different countries, the ISPD 2016 Recommendations set an international goal of a total peritonitis rate no more than 0.5 episodes per year at risk. ${ }^{7}$

\section{Peritonitis Agents}

In the literature, the most common described pathogens responsible for PD related peritonitis are aerobic bacteria, such as coagulase-negative staphylococcal species (eg, Staphylococcus epidermidis) that commonly colonize human skin and hands, and Staphylococcus aureus. Together they are responsible for $50 \%$ or more of infections in most series. ${ }^{7,8}$ Our findings corroborate the former, as indeed the most frequent agents found were Coagulase-Negative Staphylococci (23\%) and Staphylococcus aureus (20\%, with 5\% MRSA).

Antibiotics are essential in the treatment of infectious diseases; however, their widespread abuse in medical care and outside human medicine has resulted in the development of (multi)drug resistance worldwide. ${ }^{2}$ Antibiotic resistance in microorganisms in Portugal was addressed by a retrospective unicenter PD peritonitis related study (1985 - 2010; 588 episodes), which encountered a total methicillin resistance in S. epidermidis of $7 \%$ and $2 \%$ for S. aureus. ${ }^{9}$ In our unit we also observed a relative increasing emergence of more virulent pathogens per decade of study such as Pseudomonas (8\%), MRSA (5\%) and other Gram negative (8\%). This increase in frequency of methicillin-resistant agents led our nephrologists to switch the peritonitis empirical antibiotic protocol from Cefazolin/Ceftazidime to Vancomycine/Ceftazidime, following ISPD Peritonitis Recommendations $2016 .{ }^{7}$ Regarding peritonitis prophylaxis protocols, we have followed ISPD recommendations, such strategies as Staph. aureus carrier screening and treatment before catheter implantation, oral empirical antibiotic administration before the procedure as well as adoption of external orifice care guidelines, amongst others. Gram-negative bacterial infections led to the removal of the peritoneal catheter, transfer to HD or death in $49 \%$ of cases (vs. $17 \%$ in Gram-positive).

Regarding fungal peritonitis, reports vary widely, ranging from $1 \%$ to $15 \%$ of peritonitis episodes, but they are almost always associated with significant morbidity and higher mortality rate (up to $50 \%$ ). ${ }^{2,10,11}$ We only assessed 2 cases (1\%) and therefore our unit's peritonitis protocol does not include empirical antifungal treatment, despite worse outcome - both led to PD catheter removal and technique dropout.

Culture-negative peritonitis still account for up to $12 \%$ of all peritonitis episodes. ${ }^{12}$ These may be owing to recent antibiotic exposure, suboptimal sample collection or culture methods, or because of unusual organisms such as fungi. Although they are rare, a delay in the diagnosis and implementation of appropriate treatment can result in adverse patient outcomes, including death. ${ }^{2}$ We obtained a higher number of negative cultures $(n=33,15 \%)$ than the international recommendations, probably because of technical problems during sample collection and processing. Since we obtained a cure rate of $97 \%$ 
in these cases (Table II), a fungal undiagnosed etiology renders less likely. As depicted in Graphic 1, in the first decade of study we obtained negative cultures in 33\% of the cases; however, in the following two decades, this value lowered significantly to $21 \%$ and then $7 \%$. We believe that the improvement of microbiological screening method quality as well as the growing dedication of our pathology department staff throughout time, allowed a steadily decrease in these numbers.

\section{PD Modality}

In our study, APD had a protective impact for peritonitis.

Multiple risk factors for developing peritonitis have been identified, but there remains conflicting evidence on peritonitis risk between CAPD and APD in literature. ${ }^{13}$ There have been reports of lower ${ }^{14,15}$, similar $^{16-19}$, or higher ${ }^{20,21}$ peritonitis rates in patients treated with APD compared with CAPD, but the available evidence is predominantly based on observational studies, with only two small randomized controlled trials ${ }^{14,16}$ and a meta-analysis of these trials ${ }^{22}$. In a large multicenter registry study with almost 7000 patients, Patrick G. Lan et al. demonstrated that PD modality was not associated with a patient's overall risk of developing peritonitis. However, they found that APD was associated with a lower risk of culture-negative peritonitis, which was balanced by an increased risk of Gram-negative peritonitis compared with CAPD. ${ }^{13}$ Most of these studies were conducted at different time periods (raising the possibility of vintage bias), involved small patient numbers and had low peritonitis event rates. They also had different center-specific and patient-specific factors (including medical comorbidities, prior RRT, and unit practices). ${ }^{13}$ This subject is also a matter of debate in the ISPD Peritonitis Recommendations 2016, where it is assumed that the published studies that compared APD and CAPD, so far, showed conflicting results. However, most of these studies were observational rather than randomized trials. At present, the choice of APD versus CAPD should not be based on the risk of peritonitis. $^{7}$

CAPD and APD differ significantly in the frequency and method of making the connections and disconnections between the PD catheter and dialysate bags. This difference raises the question of whether one technique predisposes to or mitigates against the risk of the patient acquiring a peritoneal infection, due to contamination from repeated connections. ${ }^{23}$

The last two decades have witnessed a substantial change in PD practice. Understanding the different evolutions of the connection systems is critical when comparing peritonitis rates for PD patients during different periods. The connectology for CAPD has changed significantly over the years, from manually spiking bags with a separate connection and disconnecting with the dialysate bag and drain bag for each exchange to the twin bag systems that presently are standard. The twin bag system is the only CAPD setup available today in most parts of the world and is the dominant reason for the reduction in risk for peritonitis in individuals undergoing PD. ${ }^{23}$ In our department, this method was implemented before the study period (1993); thus all patients were treated under such conditions, therefore not altering results.
Still regarding technique evolution, another important step was related to the introduction of more biocompatible solutions, which we started using in our unit gradually (between 2001 and 2004). however we didn't have this data recorded and this variable couldn't be accessed (as we also assume it to be a limitation of our study). To mitigate this limitation, and other factors that possibly were not considered in this subject, we introduced a variable which tried to acknowledge technique evolution by splitting the time period in two halves (PD initiation period 1993 - 2004 vs. 2005 - 2016), being all patients in the former half already using these new biocompatible solutions.

\section{Impact of peritonitis}

In our research, the number of peritonitis did not correlate with an increased probability of death. However, the number of peritonitis did negatively impact technique survival, which was also worsened by peritonitis occurrence during the first treatment year, emphasizing the importance of early peritonitis prevention.

Regarding the matter of prevention, we consider that attention should be focused on the cumulative experience of the PD unit, improvement of PD technique and use of more functional equipment and more biocompatible solutions.

\section{Study limitations}

This study presents some significant limitations, including a single-center retrospective design and a relatively small size of the sample, which may have limited the consistency of the results. For instance, of the 225 included patients, only 65 were on APD, which can introduce an interpretation bias of the results.

As mentioned before, the lack of data on the evolution of the PD technique itself, such as the precise moment of introduction of more biocompatible solutions, represents a limitation of our study.

Our multivariate model considered the most prominent confounding variables already known to influence the risk for peritonitis, but limited knowledge on the factors portending this complication add some uncertainty to the reliability of the analysis.

\section{CONCLUSIONS}

In sum, APD seems to have a protector impact for peritonitis, possibly due to the less risk of contamination of fewer connections, and the number of peritonitis but also peritonitis occurrence during the first treatment year worsened technique survival.

Our unit's rate of peritonitis was found overall to be within internationally recommended values but we now emphasize to patients the importance of early (one-year) peritonitis prevention.

Disclosure of potential conflicts of interest: none declared 


\section{References}

1. Fenton SS, Schaubel DE, Desmeules M et al. Hemodialysis versus peritoneal dialysis: a comparison of adjusted mortality rates. Am J Kidney Dis. 1997;30(3):334-342.

2. Cho Y, Struijk DG. Peritoneal dialysis-related peritonitis: atypical and resistant organisms. Semin Nephrol. 2017;37(1):66-76

3. Bunke CM, Brier ME, Golper TA. Outcomes of single organism peritonitis in peritoneal dialysis: gram negatives versus gram positives in the Network 9 Peritonitis Study. Kidney Int. 1997;52(2):524-529 .

4. Guo A, Mujais S. Patient and technique survival on peritoneal dialysis in the United States: evaluation in large incident cohorts. Kidney Int Suppl. 2003; 64(88)S3-12.

5. Perez Fontan $\mathrm{M}$ et al. Peritonitis-related mortality in patients undergoing chronic peritoneal dialysis. Perit Dial Int. 2005;25(3):274-284.

6. Htay $\mathrm{H}$ et al. Center effects and peritoneal dialysis peritonitis outcomes: Analysis of a national registry. Am J Kidney Dis. 2018;71(6):814-821.

7. Li PK et al. ISPD peritonitis recommendations: 2016 update on prevention and treatment. Perit Dial Int. 2016;36(5):481-508.

8. Salzer WL. Peritoneal dialysis-related peritonitis: challenges and solutions. Int J Nephrol Renovasc Dis. 2018;11:173-186.

9. Rocha A et al. Temporal trends in peritonitis rates, microbiology and outcomes: the major clinical complication of peritoneal dialysis. Blood Purif. 2012;33(4):284-291.

10. Wang AY et al. Factors predicting outcome of fungal peritonitis in peritoneal dialysis: analysis of a 9-year experience of fungal peritonitis in a single center. Am J Kidney Dis. 2000;36(6):1183-1192.

11. Miles $R$ et al. Predictors and outcomes of fungal peritonitis in peritoneal dialysis patients. Kidney Int. 2009;76(6):622-628.

12. Fahim M et al. Culture-negative peritonitis in peritoneal dialysis patients in Australia: predictors, treatment, and outcomes in 435 cases. Am J Kidney Dis. 2010;55(4):690-697.

13. Lan PG et al. The association between peritoneal dialysis modality and peritonitis. Clin J Am Soc Nephrol. 2014;9(6):1091-1097.

14. de Fijter CW et al. Clinical efficacy and morbidity associated with continuous cyclic compared with continuous ambulatory peritoneal dialysis. Ann Intern Med 1994;120(4):264-271.
15. Sanchez AR, Madonia C, Rascon-Pacheco RA. Improved patient/technique survival and peritonitis rates in patients treated with automated peritoneal dialysis when compared to continuous ambulatory peritoneal dialysis in a Mexican PD center. Kidney Int Suppl. 2008; 73(108):S76-80.

16. Bro $S$ et al. A prospective, randomized multicenter study comparing APD and CAPD treatment. Perit Dial Int. 1999;19(6):526-533.

17. Yishak A et al. The outcome of peritonitis in patients on automated peritoneal dialysis. Adv Perit Dial. 2001;17: 205-208.

18. Akman $\mathrm{S}$ et al. Peritonitis rates and common microorganisms in continuous ambulatory peritoneal dialysis and automated peritoneal dialysis. Pediatr Int 2009;51(2):246-249.

19. Nessim SJ et al. Predictors of peritonitis in patients on peritoneal dialysis: results of a large, prospective Canadian database. Clin J Am Soc Nephrol 2009;4(7):1195-1200.

20. Golper TA et al. Risk factors for peritonitis in long-term peritoneal dialysis: the Network 9 peritonitis and catheter survival studies. Academic Subcommittee of the Steering Committee of the Network 9 Peritonitis and Catheter Survival Studies. Am J Kidney Dis. 1996;28(3):428-436.

21. Oo TN, Roberts TL, Collins AJ. A comparison of peritonitis rates from the United States Renal Data System database: CAPD versus continuous cycling peritoneal dialysis patients. Am J Kidney Dis. 2005;45(2):372-380.

22. Rabindranath KS et al. Automated vs continuous ambulatory peritoneal dialysis: a systematic review of randomized controlled trials. Nephrol Dial Transplant. 2007;22(10):2991-2998.

23. Daly $\mathrm{C}$ et al. Double bag or $\mathrm{Y}$-set versus standard transfer systems for continuous ambulatory peritoneal dialysis in end-stage renal disease. Cochrane Database Syst Rev. 2001; 13(2):CD003078.

\section{Correspondence to:}

Hospital do Espírito Santo de Évora - Serviço de Nefrologia

Largo Senhor da Pobreza

7000-811 Évora, Portugal

E-mail: davidcarvalhofiel@gmail.com 